\title{
Physical Load Affects Perceptual-Cognitive Performance of Skilled Athletes: a Systematic Review
}

\author{
M. Schapschröer ${ }^{1 *}$ (D, S. Lemez ${ }^{2}$, J. Baker ${ }^{2}$ and J. Schorer ${ }^{1}$
}

\begin{abstract}
Background: Many researchers have considered the impact of physical exercise on perceptual-cognitive performance. There have also been a substantial number of studies that have examined how perceptual-cognitive skills differ between elite athletes and non-athletes. However, the knowledge on how physical exercise interacts with perceptual-cognitive skill is limited.

This systematic review aims to provide detailed information on how athletes' perceptual-cognitive performance is influenced by acute physical exercise load and whether these effects differ between elite athletes and lesser skilled groups.

Methods: A systematic review was conducted using different combinations of the keywords physical load, acute, exercise, perception, cognition, perceptual, cognitive, sport, and athlete with the PubMed and SportDiscus databases. Additional articles were found through screening the references of these papers.

Articles had to (a) be full journal articles written in English, (b) include an athlete sample, (c) examine acute effects of physical exercise, and (d) measure a perceptual-cognitive task as the dependent variable.

Results: Twenty-six articles matched the inclusion criteria. Results suggested the impact of acute physical exercise on perceptual-cognitive performances of athletes depends on the specificity of the induced exercise and perceptual-cognitive task. Additionally, speed and accuracy were influenced differently by physical exercise. Furthermore, skilled athletes seem to be more positively influenced by acute physical exercise than novices.

Conclusion: Since many factors influence perceptual-cognitive expertise, future research should be highly precise (e.g., regarding the definition of variables, the intensity of the physical exercise) and specific (e.g., regarding the tasks used, the type of the physical exercise).
\end{abstract}

Keywords: Expertise, Acute physical exercise, Perception, Cognition, Athlete

\section{Key Points}

1. The impact of acute physical exercise on perceptualcognitive performances of athletes depends on the specificity of the induced exercise and perceptualcognitive task.
2. Acute physical exercise seems to improve expert athletes' speed in perceptual-cognitive tasks but does not affect accuracy.

3. Expert athletes are more positively influenced by acute physical exercise than lesser skilled people.

\section{Background}

Many researchers have considered the impact of physical exercise on perceptual-cognitive performance. For ex-

* Correspondence: m.schapschroeer@uni-oldenburg.de

${ }^{1}$ Institute of Sport Science, Carl von Ossietzky University Oldenburg,

Ammerländer Heerstraße 114-118, 26129 Oldenburg, Germany

Full list of author information is available at the end of the article ample, several reviews and meta-analyses have examined the influence of physical load on different aspects of cognition (e.g., [1-6]) or perception-action coupling 
(e.g., [7]). There has also been a substantial number of studies examining how perceptual-cognitive skills differ between elite athletes and non-athletes (for an overview, see [8]). However, our knowledge of how acute physical exercise influences perceptual-cognitive performance, particularly among performers at different skill levels, is limited and the results are controversial.

The impact of physical exercise on perceptualcognitive performance is, among several other factors (e.g., the type of the cognitive task induced), dependent on the type (e.g., intensity, duration) of the induced physical exercise. In his review of the effects of acute exercise on cognition, Tomporowski [6] concluded that submaximal exercise of up to $60 \mathrm{~min}$ in duration facilitates information processing, but extended exercise impairs information processing and memory functions. Prior research suggests an inverted U-relationship (arguably caused by arousal), with moderate physical exercise intensity having a positive effect on perceptual-cognitive performance, while high exercise intensity has a negative effect [9]. However, the assumption of an inverted Ueffect [9] has been criticized with several studies not supporting this hypothesis (e.g., [3, 4, 7]). Instead, it appears that different components, such as exercise intensity, duration, or task type interact and add complexity to our understanding of the impact of physical exercise on perception/cognition [3, 4, 10]. For example, Roig and colleagues [5] conducted a meta-analysis that investigated acute (29 studies) and long-term (21 studies) effects of cardiovascular exercise on human memory. The results from their review revealed that acute exercise had moderate-sized effects on short-term memory and moderate to large-sized effects on long-term memory [5]. Further, as it relates to speed and accuracy of cognition, McMorris et al. [3] revealed that acute, intermediate intensity exercise improves speed of response in working memory tasks but impairs accuracy, while no speed-accuracy trade-off was found. In another metaanalysis examining effects of differing intensities of acute exercise on cognition, McMorris and Hale [4] showed that speed and accuracy were affected differently; moderate intensity exercise produced faster speeds of processing without a significant change in accuracy. However, the authors suggested previous work may have failed to choose tests with sufficient complexity to elicit exercise-induced impacts on accuracy.

The complexity of the task is another factor that seems to be important when considering the impact of physical exercise on perceptual-cognitive performances. As McMorris and Hale [4] emphasized, complex tasks seem to be more affected by physical exercise than simple tasks. For speed, large effects of physical exercise on executive tasks were found while only small effects were shown for simple tasks [4]. For accuracy, no effects were found, regardless of the complexity of the task. In their recent meta-analysis, McMorris et al. [10] looked at the impact of physical exercise on cognition from a neurochemical perspective. Depending on the interaction of exercise, brain catecholamines (which are responsible for increases in arousal by activating the reticular formation) and cognition, McMorris et al. [10] describe three different types of tasks, differentiating between working memory tasks (e.g., Stroop color test, Simon task, switch visual attention tasks, tasks that require selecting relevant sensory information), attention/perception tasks (e.g., simple or choice reaction-time tasks with flashing lights, visual search tasks), and long-term memory tasks $[3,4,10]$. These three types of tasks seem to be differentially affected by physical exercise, which might be explained with the concentration and proportion of dopamine and adrenalin in the brain under different stress levels. While working memory tasks improve under moderate physical exercise, research indicates deterioration in these tasks during heavy exercise [10]. In contrast, attention/perception tasks show a linear improvement with increases in exercise intensity [10]. Finally, long-term memory or learning tasks seem to be especially positively influenced by heavy exercise [10].

Research investigating perceptual-cognitive expertise differences has overwhelmingly shown that experts outperform novices in several perceptual-cognitive areas, such as attention, processing speed or pattern recall and recognition tasks (e.g., $[8,11,12])$. When examining whether general or sport-specific performances differentiate between experts and non-experts, two different approaches have been examined. First, the "cognitive component skill approach" focuses on examining whether general cognitive measures capture expertise differences in sport (e.g., $[12,13])$. Some authors argue that sport training (and the experiences gained with this) is a form of cognitive training that leads to, for example, more efficient brain networks and brain plasticity, which leads to enhanced cognitive processing [12]. Furthermore, some studies have shown that a high fitness level improves cognitive functioning because of changes to structural and functional aspects of the brain [13]. This is in line with the "Cardiovascular Fitness Hypothesis" that indicates regular exercise changes brain functions, which lead to cognitive benefits (e.g., [13]). In their meta-analyses on the relationship between expertise in sport in laboratory measurements, Voss et al. [12] noted that athletes are better in general processing speed and attentional tasks. However, the authors emphasized that the results are controversial and that the "cognitive component skill approach" has often been criticized for not considering the complex environment that might also be important when examining superior expert performances in sport $[12,14]$. An alternative perspective, the 
"expert performance approach" aims to study athletes under ecologically valid and sport-specific contexts. This approach uses tasks that are representative for the specific domain of expertise and is therefore important when examining expertise differences in sport-specific perceptual-cognitive tasks $[8,11,14]$. In this context, the relatively high number of studies examining perceptualcognitive expertise differences in sport reinforces the conclusion that skilled performers process information within their domain of expertise differently than novices. For example, Mann and colleagues' [8] meta-analysis on perceptual-cognitive expertise in sport concluded that experts' performances are superior for specific measurements of response accuracy and response time, which relates to better detection and processing of perceptual cues. Furthermore, differences were found in visual search behaviors, with experts having fewer fixations of longer duration compared to non-experts [8]. However, Mann et al. [8] indicated that certain factors, such as sport type or the type of the stimulus presentation, moderated the relationship between perceptual-cognitive skills and level of expertise. With these moderators in mind, and considering the specificity of perceptual-cognitive expertise (e.g., $[15,16])$, the very small number of studies looking at the influence of different physical exercises on athletes' perceptual-cognitive performances is surprising since athletes have to combine these tasks with the specific physiological demands of their sport.

\section{Objectives}

The aim of this systematic review was to provide an overview of the existing literature on the impact of different acute physical exercises on perceptual-cognitive performances of athletes. In the context of many sports, different perceptual-cognitive skills (e.g., decisionmaking and anticipation performance or pattern recall and pattern recognition tasks (for an overview, see [8])) play important roles and have often been used to compare athletes to non-athletes. As noted earlier, two different approaches have been applied when examining experts' superiority in perceptual-cognitive tasks and results have often been controversial (e.g., [12]). However, research has overwhelmingly shown that experts are superior to novices if structured and sport-specific tasks are used [8]. Considering (a) the relatively high number of studies examining the impact of physical load in nonathletes and (b) the specificity of perceptual-cognitive expertise, this review aims to provide detailed information on how athletes' perceptual-cognitive performance is influenced by acute physical exercise load and whether these effects differ between elite athletes and lesser skilled groups.

\section{Methods}

A systematic search was conducted following the guidelines from the Preferred Reporting Items for Systematic Reviews and Meta-Analyses (PRISMA) statement for systematic review [17].

\section{Information Sources and Search Process}

The systematic search was performed using a general search engine (i.e., PubMed) and a sport-specific (i.e., SportDiscus) database. Nine combinations of keywords were included in both databases: "Physical load + perception + sport," "Physical load + cognition + sport," "Acute + exercise + perception + sport," "Acute + exercise + cognition + sport," "Physical load + perception + athlete," Physical load + cognition + athlete," "Acute + exercise + perception + athlete," "Acute + exercise + cognition + athlete," "Exercise + perceptual + cognitive." The "All Fields" search query was utilized to provide the best opportunity to capture all relevant articles for our review. The article search was completed on December 15, 2015, and a publication year cut-off was not instituted. Additionally, the references from the articles found through the database searches were screened to filter out further articles that matched the inclusion criteria.

\section{Eligibility Criteria and Study Selection}

The following inclusion criteria were instituted for this review: full journal articles (no abstracts), written in English, athlete participants, and the study had to measure a perceptual-cognitive-task as the dependent variable. Studies were included if they examined at least one group of athletes. More specifically, studies that examined "novices" and "non-athletes" but compared them to an athlete group were included in our review. Therefore, all levels of skill were included; however, studies that only looked at novices or non-athletes (i.e., no athletes tested) were excluded. The title and abstract of each article identified through the database search were screened to see whether the article matched the inclusion criteria. All studies looking at diseases or injuries as well as perceived exertion were excluded. Since this review focused on outcomes measured through performing a perceptual-cognitive task, neuroscientific studies (e.g., directly measuring brain functioning via EEG and "only" presenting data from neuroscientific measurements) were also excluded. Finally, only studies looking at acute effects of physical exercise on perceptual-cognitive performances were considered for the review.

\section{Data Items}

Articles were analyzed by several criteria to build categories and synthesize the results. Generally, articles were screened with special consideration of the perceptual-cognitive task tested, the physical exercise 
induced, the time of testing, and the participants tested. To compare and summarize results, definitions of terms were established for each of the categories. Furthermore, the methodological quality of each study was evaluated by using the Mixed Methods Appraisal Tool (MMAT), in which the score can be presented using the descriptors $* * *, \cdots$, and $* * *$ depending on the number of criteria met out of four criteria that were evaluated per category/study [18].

\section{Perceptual-Cognitive Task}

Because of the importance of specificity in the context of perceptual-cognitive expertise (e.g., $[15,16])$, the type of perceptual-cognitive task was classified as either "general" or "specific." More concretely, a general perceptual-cognitive task was a task that was not specific to the participants' domain of expertise (e.g., general reaction-time tasks without a sport-specific context). Correspondingly, a specific perceptual-cognitive task was one that was specific to the participants' domain of expertise. Tasks were classified as being specific if they included either testing in real-game situations or under contexts similar to those of real games/competitions (e.g., soccer-specific choice reaction-time tasks using videos of game situations to test soccer players). Assuming that speed and accuracy might be affected differently by acute physical exercise (e.g., [4]), studies were also classified by what performance outcomes they measured in the task.

Since the complexity of the tasks also plays an important role when examining the influence of physical exercise on perception/cognition, the perceptual-cognitive tasks were also classified as either being a "working memory task" or a "attention/perception task" [10]. Because this systematic review focused on the impact of acute physical exercise, the third category "long-term memory task" was not tested in the studies included in this review.

\section{Physical Exercise}

Since the impact of physical exercise on cognitive functioning is dependent on the characteristics of the physical exercise load (e.g., $[1,19]$ ), and considering the specificity of perceptual-cognitive expertise [15], physical exercises were also classified as general or specific. In accordance with the descriptions for the perceptualcognitive task, a general physical exercise was an exercise that was not specific for the participants' domain of expertise (e.g., exercises on a cycle ergometer for soccer players), whereas an example of a specific physical exercise includes an intermittent running protocol for soccer players.

In addition to the differences between these types of acute physical exercise, intensity also plays an important role in perceptual-cognitive performance (e.g., [20]).
Therefore, we further classified studies by the intensity of the physical exercise induced. In line with the procedure used by McMorris and Hale [4] in their metaanalysis on the effects of differing intensities of acute exercise on different aspects of cognition, and based on the classification from Borer [21], we distinguished "low," "moderate," and "high" intensities. Low intensity exercise was defined as $<40 \%$ of maximum power output $\left(W_{\max }\right)$, moderate intensity as between 40 and $79 \%$ $W_{\max }$, and high intensity as $\geq 80 \% W_{\max }$. If studies did not report values for $W_{\max }$ but for maximum oxygen uptake $\left(\mathrm{VO}_{2 \max }\right)$ or maximum heart rate $\left(\mathrm{HR}_{\max }\right)$, these values were converted by using the formulae from Arts and Kuipers [22]: $\quad \mathrm{VO}_{2 \max }=12.1+0.866 \times \% W_{\max }$, $\% \mathrm{HR}_{\max }=46.3+0.545 \times \% W_{\max }$. This is the same procedure that McMorris and Hale [4] applied. For other given values of intensity (e.g., heart rate reserve), physical exercise was compared to the studies that provided data on $W_{\max }, \mathrm{VO}_{2 \max }$, or $\mathrm{HR}_{\max }$ and were accordingly classified into one of the groups. In addition to these three classifications of exercise intensity, a fourth, "intermittent/interval exercise," was added, which alternated high- and low-intensity exercises.

\section{Participants}

To create consistent terms for the different levels of expertise, participants were classified into three comparable groups: experts, advanced, and novices. Athletes were classified as "experts" if they were highly engaged in their sports and competed at a national level or higher. The term "elite athletes" is understood as being part of this expert group. "Advanced athletes" were performers who trained regularly, in a structured way, and competed at a state, provincial, or regional level. The term athlete therefore covers all participants who trained and competed in sports at least on a regional level, regardless of the exact competition level. In the classifications created for this systematic review, the term athlete therefore covers expert as well as advanced participants. Some studies also included participants without or with very little experience in the specific sport, meaning that they did not regularly train or compete in a structured way or that they never participated in sport. These participants are classified as "novices."

\section{Risk of Bias}

All studies identified through the database search were assessed by three experts in sport expertise research. Complete agreement regarding the eligibility of each study was necessary in order for it to be included in this review. Differences between assessors were discussed until unanimity was reached. Furthermore, the methodological quality of each individual study was evaluated by using the MMAT [18]. 


\section{Results}

\section{Literature Search}

A total of 1155 articles were located through the systematic search, from which 191 duplicates were removed. An additional 18 articles were identified through searching the reference lists of the articles found through the database searches (meta-analyses and articles that matched the inclusion criteria) or through experts' suggestions. Therefore, 982 articles were screened, from which 953 were excluded after screening the title and abstract because they did not match the inclusion criteria. The main reasons for exclusion were "physical load was not induced or was not the independent variable," "perceptual-cognitive performance was not the dependent variable," "studies examining injuries or diseases," and "studies on non-athlete samples." The 29 remaining articles were checked for eligibility by reading the entire manuscript, which resulted in three more articles being excluded (i.e., were either neuroscience-based or examined a motor outcome rather than perceptualcognitive performance). An overview of the complete selection process is presented in Fig. 1. In total, 26 articles were included in the review (see Table 1).

\section{Categories}

Results were analyzed with special consideration of the perceptual-cognitive task tested, the induced physical exercise, the time of testing, and the participants included in the study. In total, 707 participants from the 26 included articles were tested, ranging from 1480 years of age. The identified articles examined a total of 194 expert, 307 advanced, and 206 novice athletes. Looking at the methodological quality of the individual studies, 23 articles reached a MMAT score [18] of three or four (75-100\% of criteria met). Thus, the overall methodological quality of the studies included in this systematic review was high.

\section{Perceptual-Cognitive Task}

Twenty-four studies used computer- or video-based tasks, with the only exceptions being Vickers and Williams [23] study which measured "quiet eye" durations in biathletes as a perceptual outcome (for more information and a review on quiet eye literature, see [24]) and the study by Elsworthy et al. [25] in which decisionmaking performance was measured in real-game situations. Most of the studies included either simple or choice reaction-time and attentional tasks in a general or specific setting (by e.g., showing sport-specific videos), with the exceptions being the studies by Vickers and Williams [23] and Casanova et al. [26] which investigated the accuracy of anticipation tasks, and Hancock and McNaughton [27] who focused on short-term memory performance.
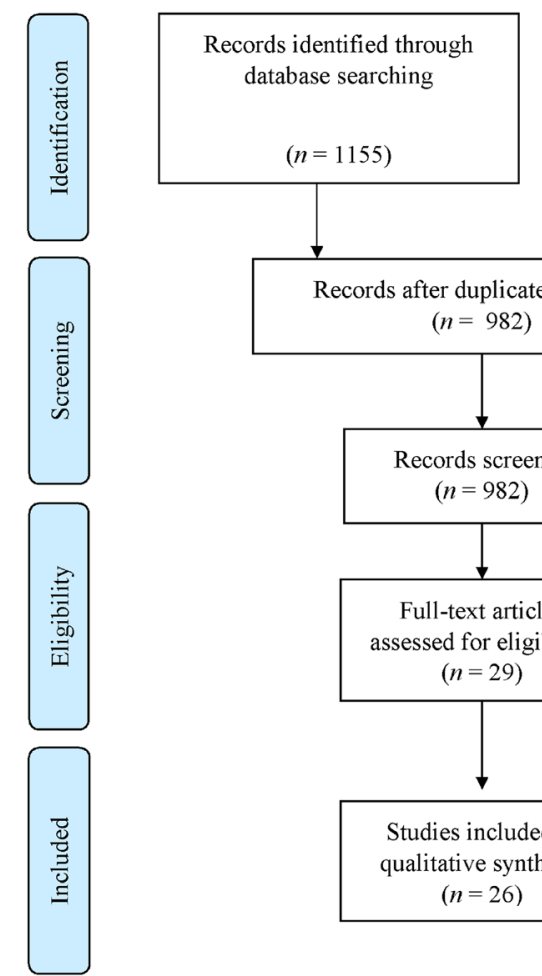

Additional records identified through other sources

$$
(n=18)
$$

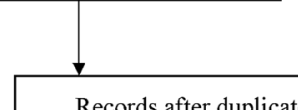

Records after duplicates removed $(n=982)$
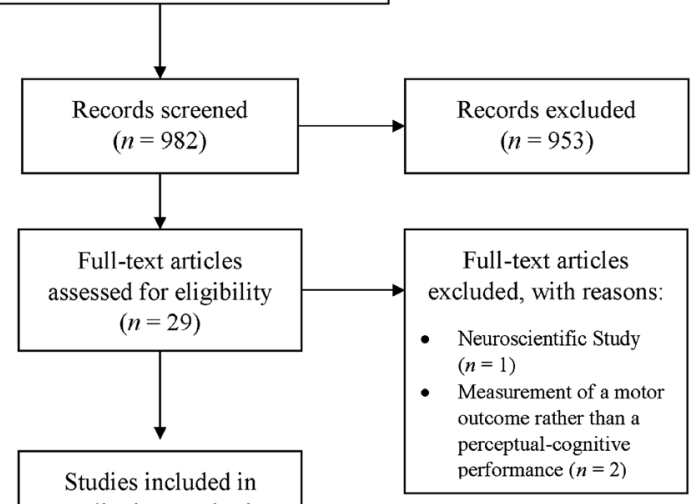

qualitative synthesis $(n=26)$

Fig. 1 PRISMA 2009 flow diagram-overview of the complete selection process. From Moher et al. [17] 


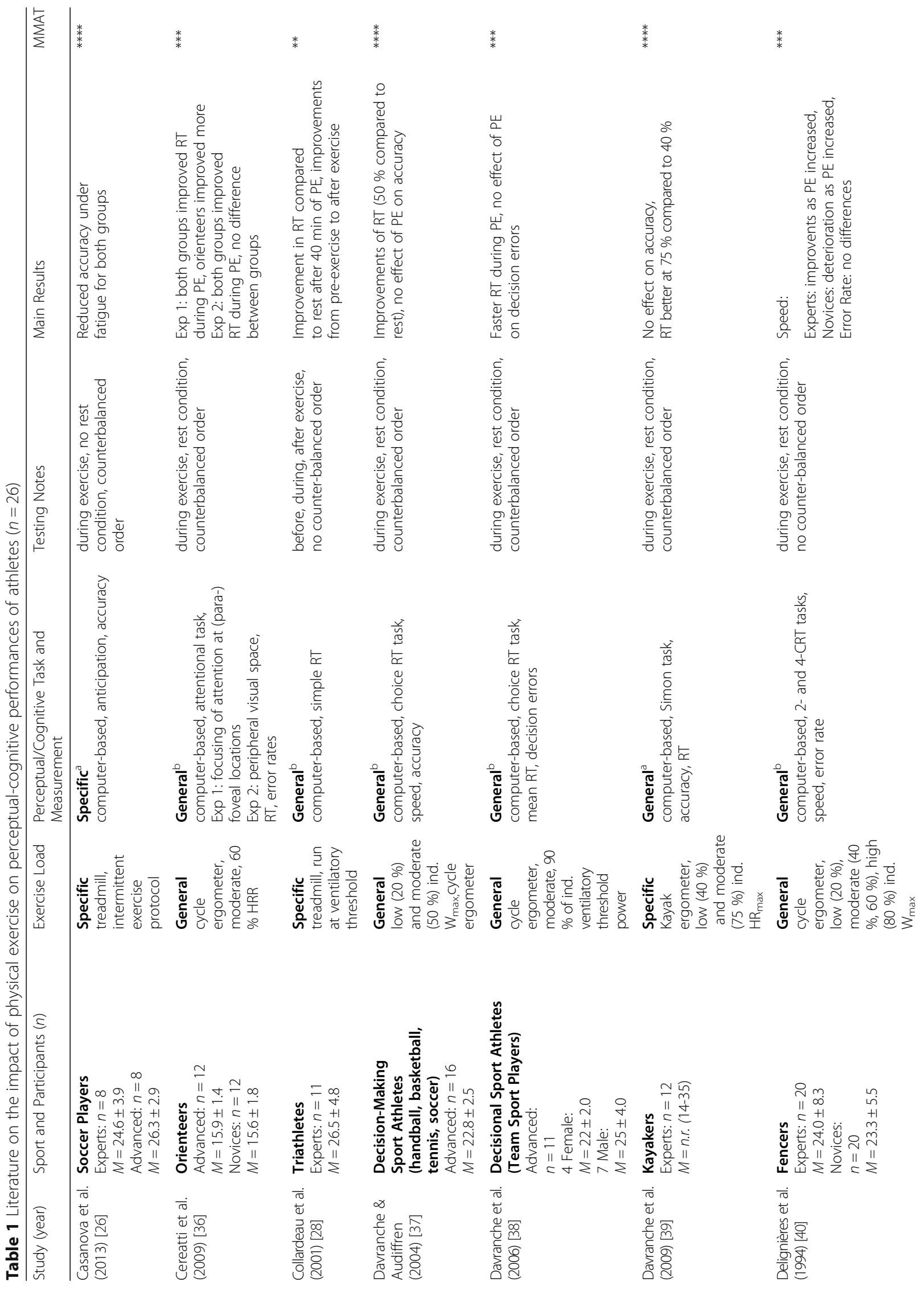




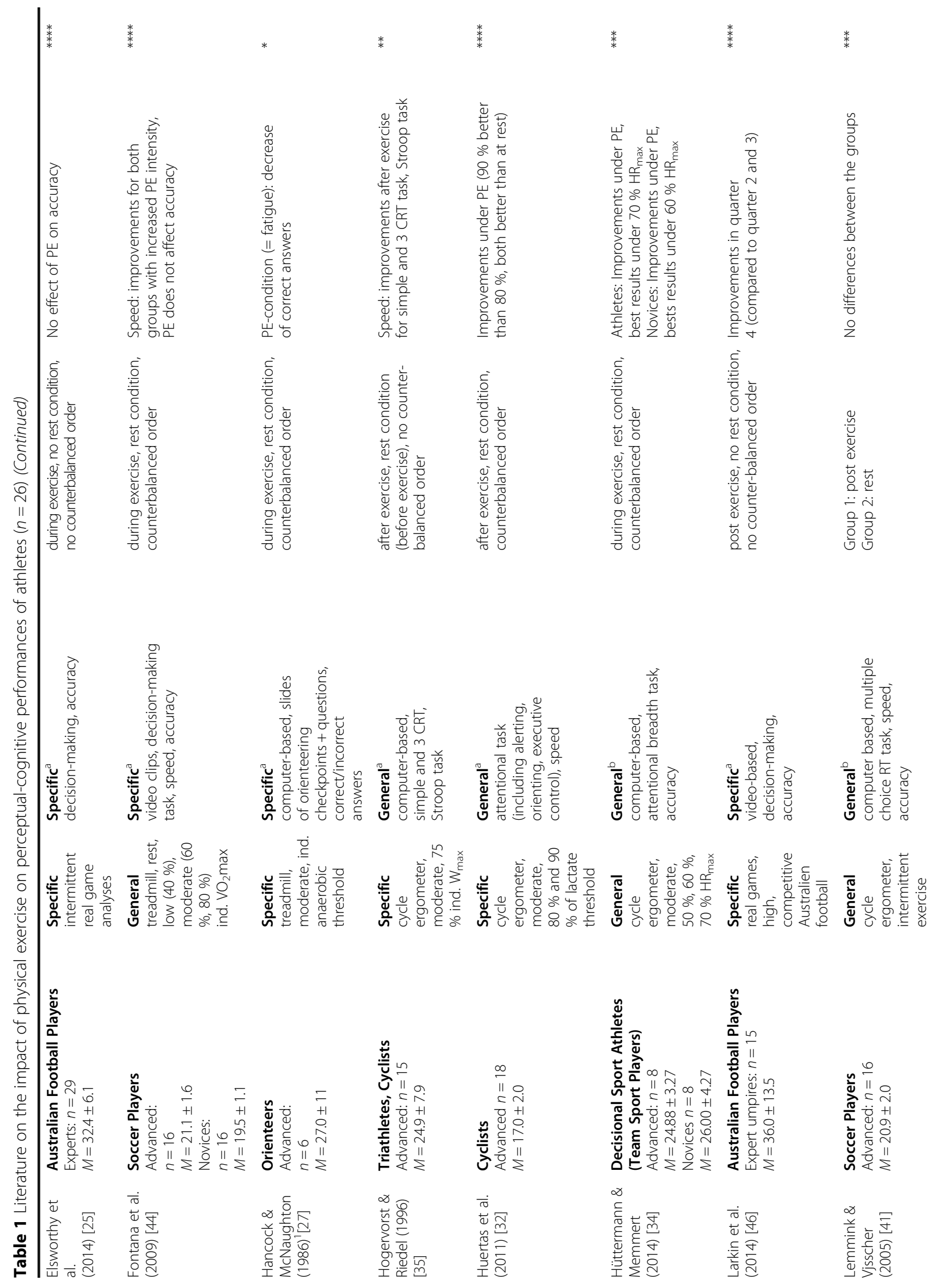




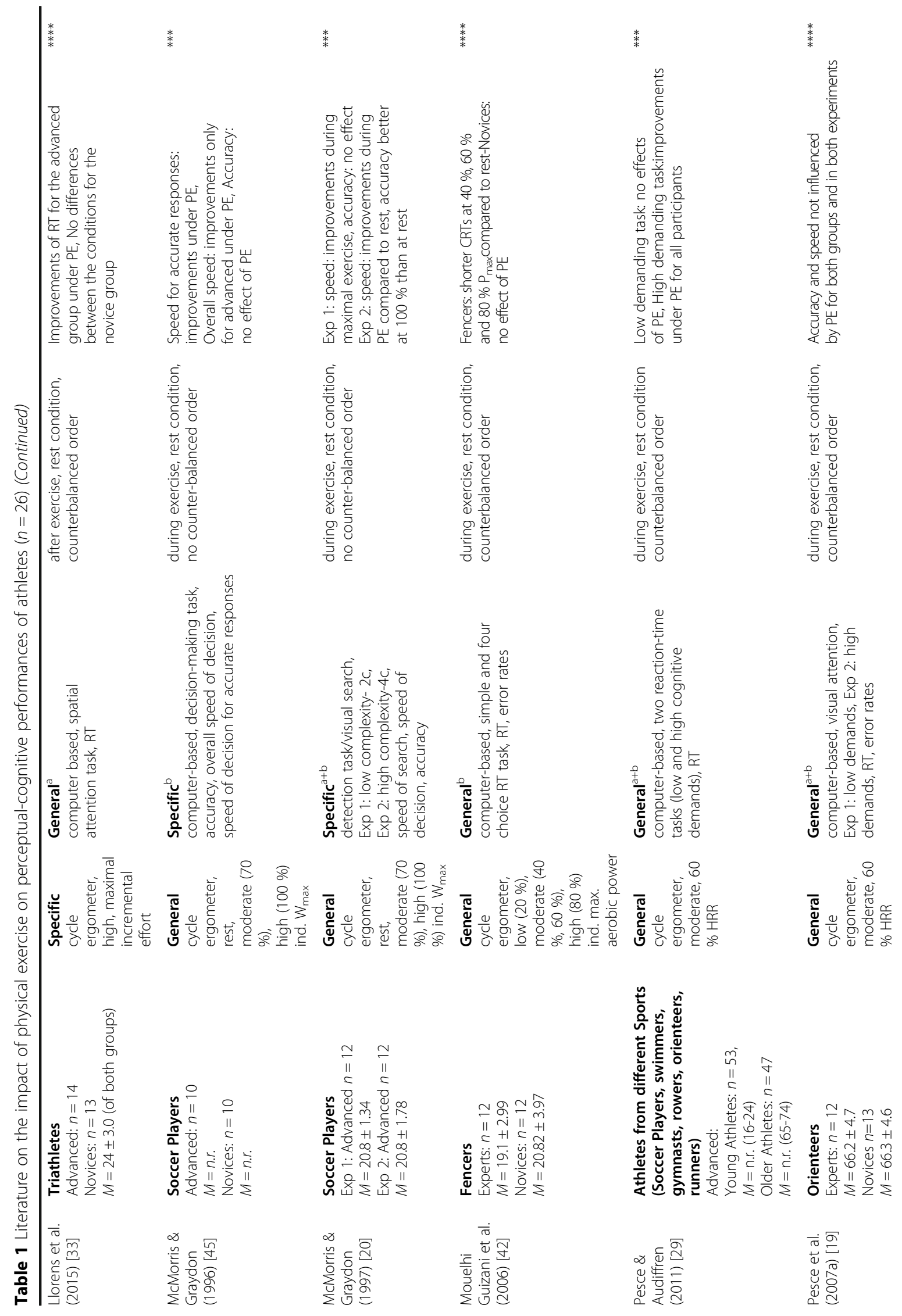




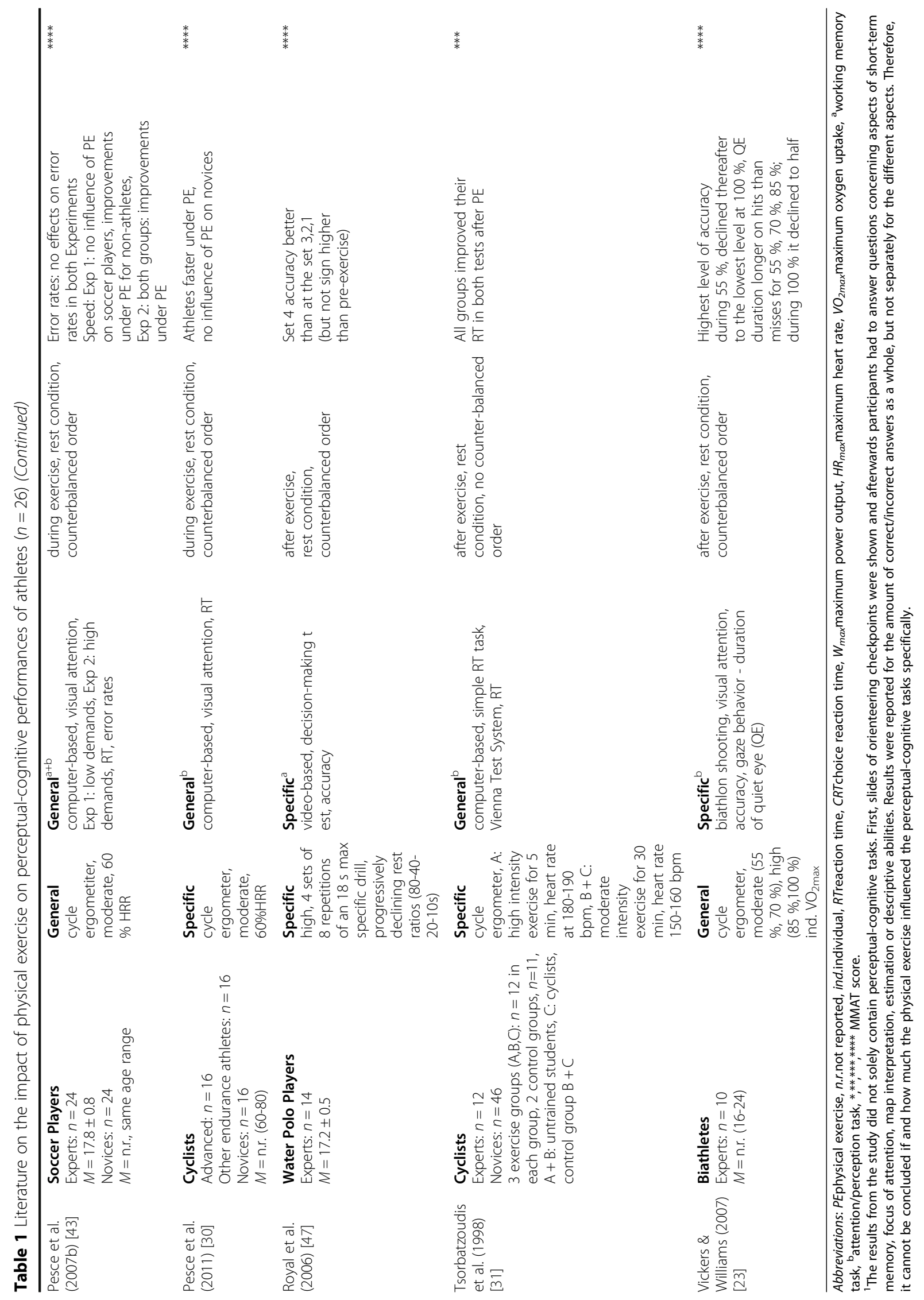


An overview of results with a focus on the specificity of the perceptual-cognitive task tested can be seen in Table 2 . From the 26 articles, 17 used a general task and nine used a specific task. Of the 17 studies that implemented a general task, six measured only reaction times [28-33], one measured only accuracy [34], and ten measured both reaction times and error rates [19, 35-43]. Fourteen of these studies revealed improvements for reaction times under acute physical exercise, while the study from Delignières et al. [40] and from Llorens et al. [33] only showed this for athlete groups. The studies from Pesce et al. [43], from Lemmink and Visscher [41] did not reveal a change in reaction times under the influence of physical exercise. Concerning the 11 studies that examined accuracy (i.e., error rates) in general perceptual-cognitive tasks, only Huttermann and Memmert [34], who did not induce any time pressure while performing an accuracy-based task, revealed improvements under physical exercise. All other studies looking at accuracy did not find any differences between conditions.

Out of the nine studies using a specific perceptualcognitive task, three considered reaction times and error rates $[20,44,45]$, five measured only accuracy [25-27, 46,47 ], and the study by Vickers and Williams [23] measured "quiet eye" durations in biathletes as a perceptual outcome. The three studies considering reaction times found an improvement under acute physical exercise. Studies of accuracy in specific perceptual-cognitive tasks displayed variable results: four studies revealed improvements [20, 23, 46, 47], two studies found deteriorations $[26,27]$, and three studies showed no effect [25, 44, 45].

Interpreting the results focusing on the types of tasks, eleven studies used a working memory task [23, 26, 27, 29, 32, 35, 39, 44-47], 11 studies attention/perception tasks [28, 30, 31, 33, 34, 36-38, 40-42], and four included both types of tasks in two experiments included in the studies $[19,20,29,43]$. The results for speed show that seven studies find improvements under physical exercise in working memory tasks $[19,20,29,32,35,39,44]$ and

Table 2 Main results for speed and accuracy of the perceptualcognitive performances based on the specificity (general or specific) of the perceptual-cognitive task

\begin{tabular}{lllll}
\hline & & + & 0 & - \\
\hline general & speed & $n=14$ & $n=2$ & $n=1$ \\
$(n=17)$ & $(n=15)$ & {$[19,28-33,35-40,42]$} & {$[41,43]$} & {$[40]$} \\
& accuracy & $n=0$ & $n=10$ & $n=1$ \\
& $(n=10)$ & & {$[19,35-43]$} & {$[34]$} \\
specific & speed & $n=3$ & $n=0$ & $n=0$ \\
$(n=9)$ & $(n=3)$ & {$[20,44,45]$} & & \\
& accuracy & $n=4$ & $n=3$ & $n=2$ \\
& $(n=9)$ & {$[20,23,46,47]$} & {$[25,44,45]$} & {$[26,27]$} \\
\hline
\end{tabular}

" + " indicates an improvement of the perceptual-cognitive performance under physical exercise, "0" no change and "-_ a deterioration
11 studies in attention/perception tasks [20, 28, 30, 31, 33, $36-38,40-42]$. No differences were found in one study in a working memory task [43] and in two studies using an attention/perception task $[19,43]$. For accuracy in working memory tasks, five studies showed improvements under physical exercise [20, 23, 45-47], five found no changes $[19,25,35,39,44]$, and three found deteriorations [26, 27, 45]. For accuracy in attention/perception tasks, two studies found improvements $[34,41]$ and six studies found no changes of perceptual-cognitive performances under physical exercise [19, 20, 28, 36-38].

\section{Physical Exercise}

An overview of results with a focus on the acute physical exercise induced can be seen in Table 3. From the 26 articles included in this review, 14 implemented a general physical exercise while 12 implemented a specific one. From the studies using a general physical exercise, six induced a moderate intensity [19, 29, 34, 36, 38, 43], two studies tested under two different conditions (a low and a moderate intensity condition) [37, 44], three studies under two conditions (moderate and high intensity) [20, $23,45]$, and two studies measured all three levels of exercise intensity in different conditions [40, 42]. Only one study [41] used an intermittent exercise, alternating a high-intensity exercise (40s) with a low-intensity exercise (20s) for $8 \mathrm{~min}$ in total. Of the 12 studies that implemented specific physical exercises, two studies induced an intermittent exercise $[25,26]$. One of these studies included two identical periods of 52 min each, each containing five low- and two high-intensity blocks [26] and the other measured performance in real-game situations, meaning that low-, moderate-, and high-intensity demands occurred [25]. In addition, five studies induced a moderate intensity exercise [27, 28, 30, 32, 35], three high intensities $[33,46,47]$, one both low and moderate intensity [39], and one both moderate and high intensity in different conditions [31].

Results for the general and specific acute physical exercise loads did not differ. Under both types, the majority of the studies reported no effect on accuracy. The number of studies showing a negative effect $[23,26,27]$ and those showing a positive effect [34, 41, 47] was equal. Differences between the perceptual-cognitive performances were observed between different exercise intensities. While no study revealed an effect for low physical exercise, the majority of studies found positive effects for moderate physical exercise on reaction times $[19,20,28-32,35-40,42,44,45]$, with the exception of the study from Pesce et al. [43] which found no effect. For accuracy, only one study showed a negative effect of moderate exercise on perceptual-cognitive performances [27] and one study found a positive effect [34], while most of the studies did not find an effect [19, 20, 36-39, 
Table 3 Results for speed and accuracy of the perceptual-cognitive performances based on the physical exercise induced

\begin{tabular}{|c|c|c|c|c|c|c|c|}
\hline \multirow[t]{2}{*}{ phys ex } & \multirow[t]{2}{*}{ intensity } & \multicolumn{3}{|l|}{ accuracy } & \multicolumn{3}{|l|}{ speed } \\
\hline & & + & 0 & - & + & o & - \\
\hline \multirow[t]{4}{*}{ general $(n=14)$} & $\begin{array}{l}\text { low } \\
n=4\end{array}$ & $n=0$ & $\begin{array}{l}n=2 \\
{[40,44]}\end{array}$ & $n=0$ & $n=0$ & $\begin{array}{l}n=3 \\
{[37,42,44]}\end{array}$ & $n=0$ \\
\hline & $\begin{array}{l}\text { moderate } \\
n=13\end{array}$ & $\begin{array}{l}n=2 \\
{[23,34]}\end{array}$ & $\begin{array}{l}n=9 \\
{[19,20,36-39,43-45]}\end{array}$ & $n=0$ & $\begin{array}{l}n=10 \\
{[19,20,29,36-38,40,42,44,45]}\end{array}$ & $\begin{array}{l}n=1 \\
{[43]}\end{array}$ & $n=0$ \\
\hline & $\begin{array}{l}\text { high } \\
n=5\end{array}$ & $n=0$ & $\begin{array}{l}n=3 \\
{[20,40,45]}\end{array}$ & $\begin{array}{l}n=1 \\
{[23]}\end{array}$ & $\begin{array}{l}n=4 \\
{[20,40,42,45]}\end{array}$ & $n=0$ & $n=0$ \\
\hline & $\begin{array}{l}\text { intermittent } \\
n=1\end{array}$ & $\begin{array}{l}n=1 \\
{[41]}\end{array}$ & $n=0$ & $n=0$ & $\begin{array}{l}n=1 \\
{[41]}\end{array}$ & $n=0$ & $n=0$ \\
\hline \multirow[t]{4}{*}{ specific $(n=12)$} & $\begin{array}{l}\text { low } \\
n=1\end{array}$ & $n=0$ & $n=0$ & $n=0$ & $n=0$ & $\begin{array}{l}n=1 \\
{[39]}\end{array}$ & $n=0$ \\
\hline & $\begin{array}{l}\text { moderate } \\
n=7\end{array}$ & $n=0$ & $\begin{array}{l}n=1 \\
{[39]}\end{array}$ & $\begin{array}{l}n=1 \\
{[27]}\end{array}$ & $\begin{array}{l}n=5 \\
{[28,30-32,35,39]}\end{array}$ & $n=0$ & $n=0$ \\
\hline & $\begin{array}{l}\text { high } \\
n=4\end{array}$ & $\begin{array}{l}n=1 \\
{[47]}\end{array}$ & $n=1$ & $n=0$ & $\begin{array}{l}n=3 \\
{[31,33,46]}\end{array}$ & $n=0$ & $n=0$ \\
\hline & $\begin{array}{l}\text { intermittent } \\
n=2\end{array}$ & $n=0$ & $\begin{array}{l}n=1 \\
{[25]}\end{array}$ & $\begin{array}{l}n=1 \\
{[26]}\end{array}$ & $n=0$ & $n=0$ & $n=0$ \\
\hline
\end{tabular}

"+" indicates an improvement of the perceptual-cognitive performance under physical exercise, "0" no change and "-" a deterioration

43-45]. Similar results were found with high-intensity exercise. The majority of studies showed positive effects on speed $[20,31,33,40,42,45,46]$, but no effect on accuracy $[20,40,45]$. The exceptions were Royal et al. [47] who found a positive effect of a high physical exercise on accuracy in perceptual-cognitive tasks, and Vickers and Williams [23], who found a negative effect under $100 \% \mathrm{VO}_{2 \max }$.

\section{Time of Testing}

There are different ways of measuring perceptualcognitive tasks in combination with physical exercise. For example, the task can either be tested during or after the physical exercise has taken place. Collardeau et al. [28] conducted both measurements, while seven studies tested the perceptual-cognitive performance after completing the physical exercise [23, 31, 33, 35, 41, 46, 47]. The remaining 18 studies had participants perform the perceptual-cognitive task during the physical exercise [19, 20, 25-27, 29, 30, 32, 34, 36-40, 42-45]. Despite these different methodological approaches, there were no obvious differences in the perceptual-cognitive performances depending on the time of testing.

\section{Participants}

From the 26 articles, 13 considered athletes from individual sports, such as triathlon [28, 33, 35], biathlon [23], cycling [30-32], orienteering [27, 36, 43], kayak [39], or fencing [40, 42]. Of the 12 studies examining team sports, six looked at soccer [19, 20, 26, 41, 44, 45], three at athletes from different team sports (e.g., handball, basketball, soccer) [34, 37, 38], two at American football $[25,46]$, and one at water polo [47]. Pesce and
Audiffren [29] examined a mixed group of participants from sports such as swimming, rowing, gymnastics, or soccer. They found no performance differences between those competing in individual or team sports. The majority of studies from both classifications (individual and team) showed a positive effect of acute physical exercise on reaction times [19, 20, 23, 28-33, 35-40, 42, 44, 45] but no effect on accuracy [19, 20, 25, 37-40, 42-45].

Fourteen studies examined athletes and compared the impact of the physical exercise under different conditions, such as rest-physical exercise or different intensities of physical exercise [20, 23, 25-29, 32, 35, 37-39, 46, 47]. Lemmink and Visscher [41] divided soccer players equally into an exercise group and a nonexercise group. Eleven studies contained a novice or control group in addition to a group of athletes and tested both groups under all conditions [19, 30, 31, 33, $34,36,40,42-45]$. Seven of these studies suggested that expert and advanced athletes are influenced by acute physical exercise differently than novices, with the higher performing athletes improving their performances under physical exercise while novices did not or less [30, $33,34,36,40,42,45]$. The other four studies reported no differences between athletes and non-athletes, which suggests that no study showed improvements for nonathletes only. Thus, the overarching finding shows a tendency of athletes to benefit more from acute physical exercise than novices.

\section{Discussion}

The aim of this review was to provide a more complete understanding of the impact of acute physical exercise on perceptual-cognitive performances of athletes. As a 
whole, the majority of studies included in this systematic review suggested that athletes' perceptual-cognitive performances in speed-related tasks are improved by moderate- and high-intensity physical exercise while accuracy is not influenced. Furthermore, results indicated that athletes' perceptual-cognitive performances are more positively influenced by physical exercise than those of non-athletes.

However, looking at the specificity of the perceptualcognitive task, results of this systematic review suggest that athletes' reaction times improved for both general and specific tasks under acute physical exercise. However, acute physical exercise seems to influence accuracy differently based on whether a general or specific task was used. Eight of the 11 studies showed that physical load did not influence athletes' accuracy in general tasks, which reflects prior research in general experimental setups where participants' reaction times improved while accuracy failed to change under physical exercise (e.g., [4]). This might be explained by either a ceiling effect or by the nature of the tasks that were used [10]. As McMorris and Hale [4] indicated, some tasks (e.g., Simon task or choice reaction-time tasks) primarily focus on testing speed of processing. Accuracy measurements are mainly meant to control participants' focus on solving the tasks and to ensure that no speed-accuracy trade-off occurs $[4,10]$. However, results for accuracy related to specific tasks were more inconsistent, with four studies showing improvements [20, 23, 46, 47], two deteriorations [26, 27], and three with no effect [25, 44, 45]. Looking at the types of tasks that were used within the nine studies that included specific perceptual-cognitive tasks, no pattern in the various tests was found. For example, decision-making tasks were used in all of the three groups of results (improvements, no differences, or deteriorations). Accordingly, there was not enough consistency in the outcomes and tests of the studies reviewed as a whole, which prevents this review from establishing any clear conclusions on the effect of acute physical exercise on specific perceptual-cognitive performances of athletes. Since the sample sizes, range of cognitive tasks, and number of studies are small, replication of these results is important. Moreover, future research should further investigate how physical exercise influences athletes' performances in accuracy using a broader range of specific perceptual-cognitive tasks. Results for the type of the task (working memory task or attention/ perception task) do not seem to provide a structured pattern. Therefore, no clear conclusion can be drawn whether athletes' perceptual-cognitive performances are also influenced differently depending on the type of the task tested, as recent research indicates (cf. $[3,4,10]$ ). A possible reason for the lack of differences between these task types might be the complexity of the tasks that were used in the studies. Although working memory tasks were examined in several studies included in this systematic review (e.g., $[19,43,44])$, it is possible that the tasks were still not complex enough, as the tasks used were less complex than, for example, tasks that include planning, abstract thinking or cognitive flexibility [4]. However, looking at the specificity of the task (general vs. specific), athletes' accuracy seems to be influenced differently depending on the specificity of the perceptualcognitive task, which is in line with prior research suggesting expertise is highly specific and may not be captured using general tests (e.g., $[48,49])$. Given these limitations regarding how physical exercise influences athletes' perceptual-cognitive performances in specific tasks, a fruitful area for further research would be to address whether specific physical exercise load influences athletes' accuracy in specific perceptual-cognitive tasks, since this seems to be different than in general tests.

Results regarding exercise intensity suggested no effect of low intensity on perceptual-cognitive task performance, while moderate to high intensities seem to increase speed but not accuracy. This is partly in line with prior research on the impact of acute physical exercise on general perceptual or cognitive tasks. Prior research suggests an inverted U-relationship, with moderate physical exercise intensity having a positive effect on perceptualcognitive performance, while high exercise intensity having a negative effect ([9], e.g., [50]). However, this inverted U-relationship has been criticized and recent research emphasizes that the effects of physical exercise on cognitive tasks are especially task-type dependent (e.g., [10]). As McMorris et al. [10] suggested, moderate physical exercise seems to influence working memory and attention/perception tasks positively while highintensity exercise improves attention/perception tasks but impairs working memory tasks. The results of this review note a positive effect for high exercise intensities for both types of tasks, reinforcing the conclusion that athletes respond differently to acute physical exercise than non-athletes. This assumption is also supported by several studies that included athlete and novice groups in their experiments [30, 33, 34, 36, 40, 42, 45]. These studies showed that athletes are more positively influenced by acute exercise than non-athletes, which might be explained by athletes' familiarity with exposure to high physiological stress accompanied by high physical and mental loads. However, it is uncertain whether the level of expertise of the athlete plays a role in this context, since the examined articles do not provide very detailed athlete experience data. While well-described for the purpose of the individual studies, the lack of a standardized classification of athletes across all of the studies limits the generalizability of certain conclusions about the role of the exact level of expertise in this context. 
Emphasizing how important a detailed definition of expertise is, Baker et al. [51] proposed a taxonomy for researchers in skill acquisition and expertise. As such, future research should aim to address how physical exercise influences perceptual-cognitive performances of athletes at different levels of skill and classify these athletes using a more sensitive classification system.

Five studies induced a specific acute physical exercise in combination with a specific perceptual-cognitive task. Interestingly, as can be seen in Fig. 2, all of the five studies looked at accuracy of perceptual-cognitive tasks and the results were inconsistent (none examined speed). While two studies found reductions in accuracy with enhanced fatigue in an anticipation [26] or memory [27] task, two studies found improvements in decisionmaking tasks [46, 47], and one study found no effect in a decision-making task [25]. This might be explained by the different types of perceptual-cognitive tasks used; however, none of the studies included in this review looked at speed when including a specific exercise and a specific task. Furthermore, data on accuracy are also relatively limited. This gap of expertise research should be addressed more specifically through examining the impact of physical exercise on perceptual-cognitive performances of athletes using more rigorous methods that measure speed and accuracy and induce a highly specific physical exercise under which a sport-specific task has to be performed.

Collectively, these results highlight the various elements of specificity as they relate to perceptual-cognitive expertise. The results concerning skill-based differences, with suggesting skilled athletes profiting more from acute physical exercise than novices, is particularly noteworthy. The need to couple the specific perceptualcognitive demands of the task with motor execution (i.e., perception-action coupling) has been widely supported in motor learning and motor control research [52].

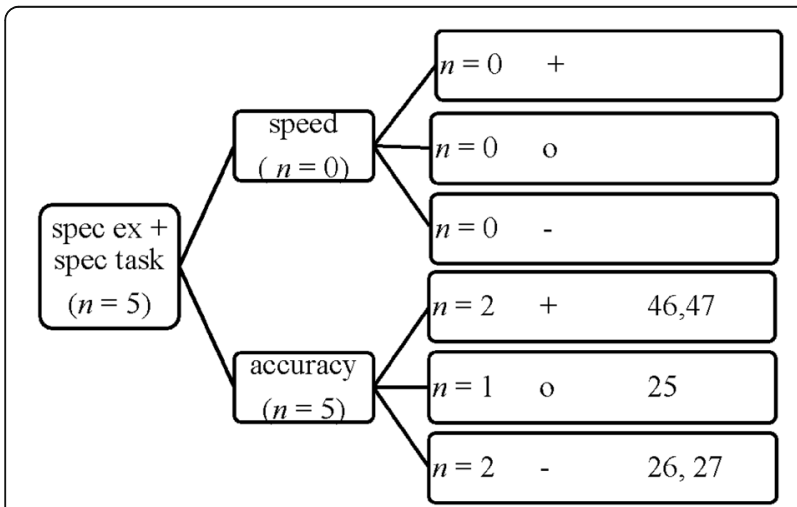

Fig. 2 Overview of results from studies inducing a specific physical exercise and testing a specific perceptual-cognitive task. "+" indicates an improvement of the perceptual-cognitive performance under physical exercise, "0" no change and "-" a deterioration
According to Gibson [53], perception and action are highly dependent on each other, and changing or manipulating one automatically influences the other [52, 53]. In the context of sport, this assumption has been supported by many studies inducing tasks where perception and action were directly coupled (e.g., $[7,54,55])$. The results of a meta-analytical investigation on effects of exercise on cognition highlight that "when perception and action are combined, the complexity of the interaction induces different effects to when cognition is detached from motor performance" ([7], p. 180). If we extend this frame of mind to the results of this systematic review, it is possible that experts, through their extensive domain-specific experience, are more accustomed to the specific coupling of acute physical exercise and perceptual-cognitive tasks. Because of their extensive practice and competitions in their field of expertise, they can handle performing a perceptualcognitive task while under high physiological stress better than less experienced participants.

Results of the current review suggest other specificityrelated parameters may also underpin the complex phenomenon of perceptual expertise. In particular, intensity-related variables may be especially important (e.g., the coupling of perception-action and intensity). While this review focused on intensity in regard to physiological stress, it is likely that intensity in this context reflects a broad term covering a range of factors related to the specificity of the measurement environment (e.g., physical load, the combination of physical and cognitive load, performing under time pressure, as well as performance-related emotions such as nervousness and competition anxiety). Prior research in sport expertise and applied sport psychology revealed that experts are superior to non-experts in several different domains of expertise, such as controlling and regulating emotions effectively (for an overview see [56]). The term perception-action-intensity coupling may help to explain expertise differences found in this systematic review as well as in other domains. Although this notion has some intuitive appeal, additional work is clearly necessary to determine the validity of this argument.

This systematic review focused on perceptualcognitive performance, which is very important when athletes actually execute their specific sport. However, to fully capture, explain, and understand the impact of physical exercise on perceptual-cognitive expertise, it is necessary that future research also considers the underlying mechanisms. Several authors have looked at the neurophysiological basis of perceptual-cognitive performance and have shown that catecholamins in the brain lead to different levels of arousal when under physical exercise, which influences the performances of perceptual-cognitive tasks $[4,7,10]$. Future research 
should extend this work by examining athletes of different levels of expertise.

\section{Limitations of the Current Review}

The current review provides an important synthesis of how acute exercise loads affect perceptual-cognitive performance; however, there were several notable limitations. One is directly connected to the specificity of perceptualcognitive expertise. Clearly, specificity is important in the context of expertise; however, we had to create broad categories in order to adequately summarize and combine results to provide an overview of the existing literature on the impact of different acute physical exercises on perceptual-cognitive performances of athletes. Although we believe this was a reasonable approach to examining the research questions addressed in this review, this may have eliminated some specific results of individual studies. It is possible that some of these detailed results that were not highlighted because of the broad categories we had to create might turn out to be important once more research in this area has been conducted. For example, the methodology applied in this review classified the physical exercise intensity induced using $W_{\max }, \mathrm{VO}_{2 \max }$, or $\mathrm{HR}_{\max }$. For other given values of intensity (e.g., heart rate reserve), physical exercise was compared to the studies that provided data on $W_{\max }, \mathrm{VO}_{2 \max }$, or $\mathrm{HR}_{\max }$ and the studies were accordingly classified into one of the groups. As McMorris and Hale [4] emphasized, this strategy contains a component of subjectivity.

Second, it was difficult to account for the different methodologies of the 25 articles included in this review. For example, not all of the studies tested the different exercise and rest conditions in a counterbalanced order (e.g., [20, 35, 44-46]). Therefore, it is not clear whether the found changes in perceptual-cognitive performances occurred due to the impact of the physical exercise induced or because of, for example, learning effects or familiarization with the task. Moreover, some studies included different exercise intensity conditions but no rest condition $[25,26,46]$. These studies, therefore, give an insight in how perceptual-cognitive performances change under different exercise intensities but do not allow us to draw any conclusions about a comparison to the performance at rest. In addition to the different methodologies applied, care should be taken when comparing and summarizing results from the different studies because of the different sample sizes and power effects across the studies. Since both are important factors when evaluating the validity and significance of results (e.g., [57]), it should be noted that there was considerable variability in the sample sizes between the different studies included in this review. For example, Hancock and McNaughton [27] examined six participants and Vickers and Williams [23] examined ten participants, whereas Pesce and Audiffren [29] included 100 participants in their study. All of the other studies had sample sizes within this range (i.e., 6 to 100 participants).

Third, this review contained a relatively large age range (between 14 and 80 years) of participants. Since this review did not focus on differences between age groups, this factor was not considered. However, the impact of physical exercise on perceptual-cognitive performances across age groups is an interesting field of further research.

Fourth, there was a disproportion in the types of sports presented in the 26 articles included in this review. Although the distribution of individual (13 studies) and team sports (12 studies) was similar (one study included athletes from different types of sports), a closer look at the sports within these categories revealed a disproportionate focus on soccer in the team sport category (six of 12 studies). Future research should therefore look at the impact of physical exercise on specific perceptualcognitive tasks across a more diverse range of sports. Looking at this field of research from different perspectives and different sports may provide important information for the field of expertise and help apply this knowledge to specific sport contexts.

Finally, one of the inclusion criteria in this systematic review was that the articles had to be written in English. Since English is the most common language in this field of research and most important articles are (also) published in English, this is in adequate method for conducting a systematic review. However, it is possible that important studies relevant to this review may exist in other languages.

\section{Conclusions}

Results of this review suggest acute physical exercise has an influence on the perceptual-cognitive performance of athletes. Moderate and high physical exercise improves speed in perceptual-cognitive tasks and does not influence accuracy in the majority of studies. Athletes seem to be more positively influenced by acute physical exercise than non-athletes. However, specificity plays an important role in the science of expertise, both in understanding the limitations of perceptual-cognitive skill and in designing valid and reliable tasks to measure these qualities. As a result, future research should examine these effects using more specific testing. For example, inducing an intermittent interval exercise for a particular team sport and testing specific perceptualcognitive performances under real-game situations, whether during or in between competition, would be valuable for future researchers and sport practitioners to explore. While this review sums up the main findings of the impact of acute physical exercise on perceptualcognitive expertise, care should be taken when making general conclusions. Since many factors influence 
perceptual-cognitive expertise and many factors interact with one another [10], it is unclear how much each single factor influences the outcome (e.g., is it the type of the physical exercise itself or a combination of the timing of the testing and the specific participants tested?). This leads to a clear need for future research that is highly precise and specific, such as when defining the expertise level of participants, choosing a specific physical exercise and a specific perceptual-cognitive task.

\section{Authors' Contributions}

The study was planned by MS, SL, and JB. MS conducted the literature search and wrote the initial draft of the manuscript. MS, SL, JB, and JS discussed and revised the manuscript together and gave final approval for publication.

\section{Competing Interests}

Marlen Schapschröer, Srdjan Lemez, Joseph Baker, and Jörg Schorer declare that they have no potential conflicts of interest and none of the authors have any competing interests in the manuscript.

\section{Ethics Approval and Consent to Participate}

This manuscript does not contain clinical studies or patient data. No financial support was received for the conduct of this study or preparation of this manuscript.

\section{Author details}

'Institute of Sport Science, Carl von Ossietzky University Oldenburg, Ammerländer Heerstraße 114-118, 26129 Oldenburg, Germany. ${ }^{2}$ School of Kinesiology and Health Science, York University, Toronto, Canada.

\section{Received: 5 April 2016 Accepted: 26 August 2016}

\section{Published online: 12 September 2016}

\section{References}

1. Brisswalter J, Collardeau M, René A. Effects of acute physical exercise characteristics on cognitive performance. Sports Med. 2002;32(9):555-66

2. Lambourne $K$, Tomporowski P. The effect of exercise-induced arousal on cognitive task performance: a meta-regression analysis. Brain Res. 2010;1341: 12-24. doi:10.1016/j.brainres.2010.03.091.

3. McMorris T, Sproule J, Turner A, Hale BJ. Acute, intermediate intensity exercise, and speed and accuracy in working memory tasks: a metaanalytical comparison of effects. Physiol Behav. 2011;102(3):421-8.

4. McMorris T, Hale BJ. Differential effects of differing intensities of acute exercise on speed and accuracy of cognition: a meta-analytical investigation. Brain Cogn. 2012:80(3):338-51. doi:10.1016/j.bandc.2012.09.001.

5. Roig M, Nordbrandt S, Geertsen SS, Nielsen JB. The effects of cardiovascular exercise on human memory: a review with meta-analysis. Neurosci Biobehav Rev. 2013;37(8):1645-66.

6. Tomporowski PD. Effects of acute bouts of exercise on cognition. Acta Psychol (Amst). 2003;112(3):297-324

7. McMorris T, Hale BJ, Corbett J, Robertson K, Hodgson Cl. Does acute exercise affect the performance of whole-body, psychomotor skills in an inverted-U fashion? A meta-analytic investigation. Physiol Behav. 2015;141: 180-9. doi:10.1016/j.physbeh.2015.01.010.

8. Mann D, Williams AM, Ward P, Janelle CM. Perceptual-cognitive expertise in sport: a meta-analysis. J Sport Exerc Psychol. 2007;29(4):457-78.

9. Yerkes RM, Dodson JD. The relation of strength of stimulus to rapidity of habit-formation. J Comp Neurol Psych. 1908;18:459-82.

10. McMorris T, Turner A, Hale BJ, Sproule J. In: McMorris T, editor. Beyond the catecholamines hypothesis for an acute exercise-cognition interaction: a neurochemical perspective. Exercise-cognition interaction: neuroscience perspectives. New York: Academic; 2016. p. 65-104

11. Starkes J, Ericsson KA. Expert performance in sports. Champaign. IL: Human Kinetics; 2003

12. Voss MW, Kramer AF, Basak C, Prakash RS, Roberts B. Are expert athletes expert' in the cognitive laboratory? A meta-analytic review of cognition and sport expertise. Appl Cogn Psychol. 2010;24(6):812-26.
13. Colcombe S, Kramer A. Fitness effects on the cognitive function of older adults a meta-analytic study. Psychol Sci. 2003:14(2):125-30.

14. Ericsson KA. How the expert performance approach differs from traditional approaches to expertise in sports: in search of a shared theoretical framework for studying expert performance. In: Starkes J, Ericsson KA, editors. Expert performance in sport: recent advances in research on sport expertise. Champaign, IL: Human Kinetics; 2003. p. 371-401.

15. Loffing F, Schorer J, Hagemann N, Baker J. On the advantage of being lefthanded in volleyball: further evidence of the specificity of skilled visual perception. Atten Percep Psychophys. 2012;74(2):446-53.

16. Williams AM, Ward JD, Ward P, Smeeton NJ. Domain specificity, task specificity, and expert performance. Res Q Exerc Sport. 2008;79(3):428-33.

17. Moher D, Liberati A, Tetzlaff J, Altman DG, Group TP. Preferred Reporting Items for Systematic Reviews and Meta-Analyses: the PRISMA Statement. PLoS Med. 2009:6(7), e1000097. doi:10.1371/journal.pmed.1000097.

18. Pluye P, Robert E, Cargo M, Bartlett G, O'Cathain A, Griffiths F, et al. Proposal: a mixed methods appraisal tool for systematic mixed studies reviews. Montréal: McGill University; 2011. p. 1-8.

19. Pesce C, Tessitore A, Casella R, Pirritano M, Capranica L. Focusing of visual attention at rest and during physical exercise in soccer players. J Sports Sci. 2007:25(11):1259-70. doi:10.1080/02640410601040085.

20. McMorris T, Graydon J. The effect of exercise on cognitive performance in soccer-specific tests. J Sports Sci. 1997;15(5):459-68.

21. Borer KT. Exercise endocrinology. Champaign: Human Kinetics; 2003

22. Arts FJ, Kuipers $\mathrm{H}$. The relation between power output, oxygen uptake and heart rate in male athletes. Int J Sports Med. 1994;15(5):228-31. doi:10.1055/ s-2007-1021051.

23. Vickers JN, Williams AM. Performing under pressure: the effects of physiological arousal, cognitive anxiety, and gaze control in biathlon. J Motor Behav. 2007:39:381-94. doi:10.3200/JMBR.39.5.381-394.

24. Rienhoff R, Tirp J, Strauss B, Baker J, Schorer J. The 'quiet eye' and motor performance: a systematic review based on Newell's constraints-led model. Sports Med. 2015. doi:10.1007/s40279-015-0442-4.

25. Elsworthy N, Burke D, Scott BR, Stevens CJ, Dascombe BJ. Physical and decision-making demands of Australian football umpires during competitive matches. J Strength Cond Res. 2014;28(12):3502-7. doi:10.1519/jsc. 0000000000000567.

26. Casanova F, Garganta J, Silva G, Alves A, Oliveira J, Williams AM. Effects of prolonged intermittent exercise on perceptual-cognitive processes. Med Sci Sports Exerc. 2013:45(8):1610-7. doi:10.1249/MSS.0b013e31828b2ce9.

27. Hancock S, McNaughton L. Effects of fatigue on ability to process visual information by experienced orienteers. / Effets de la fatigue sur la capacite a traiter les informations visuelles de pratiquants experimentes de la course d' orientation. Percept Motor Skills. 1986;62(2):491-8.

28. Collardeau M, Brisswalter J, Audiffren M. Effects of a prolonged run on simple reaction time of well trained runners. PMS. 2001;93(3):679-89.

29. Pesce C, Audiffren M. Does acute exercise switch off switch costs? A study with younger and older athletes. J Sport Exerc Psychol. 2011;33(5):609-26.

30. Pesce C, Cereatti L, Forte R, Crova C, Casella R. Acute and chronic exercise effects on attentional control in older road cyclists. Gerontology. 2011;57(2): 121-8. doi:10.1159/000314685

31. Tsorbatzoudis H, Barkoukis V, Danis A, Grouios G. Physical exertion in simple reaction time and continuous attention of sport participants. PMS. 1998; 86(2):571-6. doi:10.2466/pms.1998.86.2.571.

32. Huertas F, Zahonero J, Sanabria D, Lupiánez J. Functioning of the attentional networks at rest vs. during acute bouts of aerobix exercise. J Sport Exerc Psychol. 2011;33:649-85.

33. Llorens F, Sanabria D, Huertas F. The influence of acute intense exercise on exogenous spatial attention depends on physical fitness level. Exp Psychol. 2015;62(1)9-21.

34. Huttermann S, Memmert D. Moderate movement, more vision: effects of physical exercise on inattentional blindness. Perception. 2012;41(8):963-75.

35. Hogervorst E, Riedel W. Cognitive performance after strenuous physical exercise. Percept Motor Skills. 1996:83(2):479.

36. Cereatti L, Casella R, Manganelli M, Pesce C. Visual attention in adolescents: facilitating effects of sport expertise and acute physical exercise. Psychol Sport Exerc. 2009;10(1):136-45. http://dx.doi.org/10.1016/j.psychsport.2008. 05.002.

37. Davranche K, Audiffren M. Facilitating effects of exercise on information processing. J Sports Sci. 2004:22(5):419-28. doi:10.1080/ 02640410410001675289 
38. Davranche K, Audiffren M, Denjean A. A distributional analysis of the effect of physical exercise on a choice reaction time task. J Sports Sci. 2006;24(3): 323-9. doi:10.1080/02640410500132165.

39. Davranche K, Paleresompoulle D, Pernaud R, Labarelle J, Hasbroucq T. Decision making in elite white-water athletes paddling on a kayak ergometer. J Sport Exerc Psychol. 2009;31(4):554-65.

40. Delignières $D$, Brisswalter J, Legros P. Influence of physical exercise on choice reaction time in sports experts: the mediating role of resource allocation. J Hum Mov Stud. 1994;27(4):173-88.

41. Lemmink KAPM, Visscher C. Effect of intermittent exercise on multiple-choice reaction times of soccer players. Percept Motor Skills. 2005;100(1):85-95.

42. Mouelhi Guizani S, Bouzaouach I, Tenenbaum G, Ben Kheder A, Feki Y, Bouaziz M. Simple and choice reaction times under varying levels of physical load in high skilled fencers. J Sports Med Phys Fitness. 2006;46(2): 344-51.

43. Pesce C, Cereatti L, Casella R, Baldari C, Capranica L. Preservation of visual attention in older expert orienteers at rest and under physical effort. J Sport Exerc Psychol. 2007;29(1):78.

44. Fontana FE, Mazzardo O, Mokgothu C, Furtado Jr O, Gallagher JD. Influence of exercise intensity on the decision-making performance of experienced and inexperienced soccer players. J Sport Exerc Psychol. 2009;31(2):135-51.

45. McMorris T, Graydon J. The effect of exercise on the decision-making performance of experienced and inexperienced soccer players. Res Q Exerc Sport. 1996;67(1):109-14. doi:10.1080/02701367.1996.10607933.

46. Larkin P, O'Brien B, Mesagno C, Berry J, Harvey J, Spittle M. Assessment of decision-making performance and in-game physical exertion of Australian football umpires. J Sports Sci. 2014;32(15):1446-53. doi:10.1080/02640414. 2014.896998.

47. Royal KA, Farrow D, Mujika I, Halson SL, Pyne D, Abernethy B. The effects of fatigue on decision making and shooting skill performance in water polo players. J Sports Sci. 2006;24(8):807-15. doi:10.1080/02640410500188928.

48. Abernethy B, Neal RJ, Koning P. Visual perceptual and cognitive differences between expert, intermediate, and novice snooker players. Appl Cogn Psychol. 1994;8(3):185-211.

49. Williams AM, Burwitz K. Advance cue utilization in soccer. In: Reilly T, Clarys J, Stibbe A, editors. Science and Football, vol. 3. London, GB: E\&FN Spoon; 1993. p. 239-43.

50. Davey C. Physical exertion and mental performance. Ergonomics. 1973;16(5): 595-9.

51. Baker J, Wattie N, Schorer J. Defining expertise. A taxonomy for researchers in skill acquisition and expertise. In: Baker J, Farrow D, editors. Routledge Handbook of Sport Expertise. London: Routledge; 2015. p. 145-55.

52. Farrow D, Abernethy B. Do expertise and the degree of perception-action coupling affect natural anticipatory performance? Perception. 2003;32(9): $1127-39$.

53. Gibson JJ. An ecological approach to visual perception. Boston, MA Houghton-Mifflin; 1979.

54. Vignais N, Bideau B, Craig C, Brault S, Multon F, Kulpa R. Virtual environments for sport analysis: perception-action coupling in handball goalkeeping. IJVR. 2009:8(4):43-8.

55. Williams AM, Ward P, Smeeton NJ, Allen D. Developing anticipation skills in tennis using on-court instruction: perception versus perception and action. J Appl Sport Psychol. 2004;16(4):350-60.

56. Janelle $\mathrm{CM}$, Hillman $\mathrm{CH}$. Experts performance in sports: current perspectives and critical issues. In: Starkes JL, Ericsson KA, editors. Expert performance in sports: Advances in research on sport expertise. Champaign, IL: Human Kinetics; 2003. p. 219-50

57. Sabo R, Boone E. Statistical research methods. A guide for non-statisticiants. New York: Springer; 2013.

\section{Submit your manuscript to a SpringerOpen ${ }^{\circ}$ journal and benefit from:}

- Convenient online submission

- Rigorous peer review

- Immediate publication on acceptance

- Open access: articles freely available online

- High visibility within the field

- Retaining the copyright to your article

Submit your next manuscript at $\gg$ springeropen.com 